\title{
Hypervelocity impact properties of graphene armor via molecular dynamics simulations
}

\author{
H. Shang and W. Wang \\ Laboratory for Shock Wave and Detonation Physics, Institute of Fluid Physics, China Academy of Engineering \\ Physics, Mianyang 621900, Sichuan, China
}

\begin{abstract}
Hypervelocity impact properties of two different graphene armor systems are investigated using molecular dynamics simulations. One system is the so-called spaced armor which consists of a number of graphene plates spaced certain distance apart. Its response under normal impact of a spherical projectile is studied, focusing on the effect of the number of graphene monolayers per plate (denoted by $n$ ) on the penetration resistance of the armor. We find that under normal impact by a spherical projectile the penetration resistance increases with decreasing number of monolayers per plate $(n)$, and the best penetration resistance is achieved in the system with one graphene layer for each plate. Note that the monolayers in all the simulated multilayer graphene plates were AB-stacked. The second system being studied is the laminated copper/graphene composites with the graphene layers inside copper, on impact or back surface, or on both the impact and back surfaces. The simulation results show that under normal impact by a spherical projectile the laminated copper/graphene composite has much higher penetration resistance than the monolithic copper plate. The best efficiency is achieved when the graphene layers are on both the impact and back surfaces.
\end{abstract}

\section{Introduction}

Graphene is an allotrope of carbon, whose structure is oneatom-thick planar sheets of $\mathrm{sp}^{2}$-bonded carbon atoms that are densely packed in a honeycomb crystal lattice. It has drawn great attention since it was fabricated in the laboratory $[1,2]$. It has extraordinary mechanical, thermal, optical, and electrical properties. Lee et al. [3] measured the elastic properties and breaking strength of free-standing monolayer graphene by nanoindentation in an atomic force microscope. Their results indicated that the breaking strength is $42 \mathrm{~N} / \mathrm{m}$, corresponding to a Young's modulus of $\mathrm{E}=1.0 \mathrm{TPa}$, which establish graphene as the strongest material ever measured. Jiang et al. [4] investigated the Young's modulus of graphene by molecular dynamics simulations. Their results show that Young's modulus of graphene increases from 0.95 to $1.1 \mathrm{TPa}$ as temperature increases from 100 to $500 \mathrm{~K}$. Frank et al. [5] measured the effective spring constants of stacks of suspended graphene sheets (thickness between 2 and $8 \mathrm{~nm}$ ) using atomic force microscope. From the measured spring constants they estimated a Young's modulus of $0.5 \mathrm{TPa}$.

Using spaced plates instead of a monolithic one to increase the penetration resistance of civil and military ballistic protection systems has being investigated for a long time. But there is no consensus in this area. Dey et al. [6] studied the ballistic perforation resistance of double-layered steel plates impacted by blunt and ogival projectiles. They found that in the case of blunt projectiles a large gain in the ballistic limit is offered by doublelayered systems, but the advantages disappeared when ogival projectiles are used. Alavi Nia et al. [7] studied the perforation of single and three layered metallic targets by hemispherical-nosed cylindrical projectiles experimentally. Their results show that the single layer targets have greater ballistic limit velocities than multi-layered targets.
Mixing one material into another to enhance the mechanical properties of the latter has being studied for a long time. Yadav et al. [8] investigated the penetration resistance of laminated ceramic/polymer structures. Their experimental results illustrate that penetration resistance of an pristine ceramic structure could be improved significantly by laminating ceramic tiles with thin polymer layers in between. Makeev et al. [9] investigated the hypervelocity impact protection properties of carbonnanotube-reinforced amorphous silicon carbide composites by molecular dynamics simulations. They found that the presence of carbon nanotubes cause significant damping of the impact-induced shock-wave velocity, hence improved the impact protection properties of silicon carbide.

Since graphene has so extraordinary mechanical properties, we attempt to investigate whether it can be used in spaced armor systems or can be mixed into other material to form some kind of stronger composites. In this letter, we use molecular dynamics (MD) simulations to investigate the penetration resistance of spaced multilayer graphene plates and the laminated copper/graphene composites.

\section{Simulation methods}

We perform molecular dynamics simulations using largescale atomic/molecular massively parallel simulator (LAMMPS) package [10]. The interaction between carbon atoms in graphene is calculated using the adaptive intermolecular reactive empirical bond-order (AIREBO) potential [11], which enables simulations on bond breaking, bond reformation and failure of graphene. Copper atoms are described using the embedded atom method (EAM) [12]. The carbon-copper interaction is modeled using a Lennard-Jones form pairwise potential $V(r)=$ $4 \varepsilon\left[(\sigma / r)^{12}-(\sigma / r)^{6}\right]$. The parameters $\varepsilon=0.0328 \mathrm{eV}$ and 


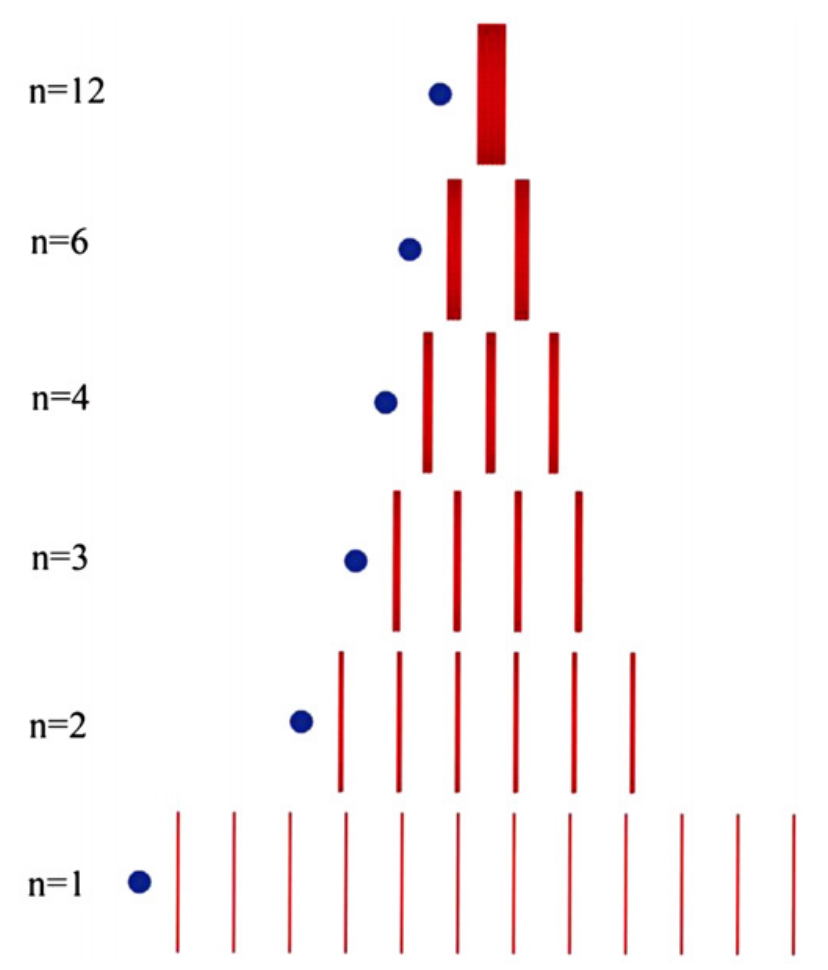

Fig. 1. Simulation models for spaced multilayer graphene plates. The sphere on the left is the projectile, and the plates on the right are the graphene plates ( $n$ denotes the number of graphene monolayers per plate).

$\sigma=2.853 \AA$ are simply averaged from those parameters for carbon-carbon and copper-copper systems [13].

\section{Results and discussion}

To compute the impact protection properties of spaced multilayer graphene plates, a serial of target models have been established. As shown in figure 1, each model contains totally 12 graphene monolayers divided into several spaced plates. The number of graphene monolayers per plate is denoted by $n$, and the distance between adjacent graphene layers is $3.35 \AA$ (that is the distance between adjacent layers in graphite). Each graphene monolayer is circular in shape with a radius of $100 \AA$ which contains about 12,000 carbon atoms. And note that the monolayers in all the simulated multilayer graphene plates were $\mathrm{AB}$ stacked. A spherical diamond cluster with a radius of $15 \AA$ (totally 2486 carbon atoms) is used as the projectile. And it is fixed rigid with purely repulsive interactions with the target. In our simulations, the boundary of the graphene sheets (in XY plane) is fixed not to move toward any directions. The time integration algorithm we used is the velocity-Verlet time stepping scheme with a time step of $0.5 \mathrm{fs}$. The system is first relaxed for $10000 \mathrm{MD}$ steps at a constant temperature of $300 \mathrm{~K}$ using NVT ensemble. After relaxation the projectile was given an appropriate initial velocity perpendicular to the target, and the subsequent evolution was calculated using an NVE ensemble.

We calculated the ballistic limit velocity for each system using the same projectile. The ballistic limit ve-

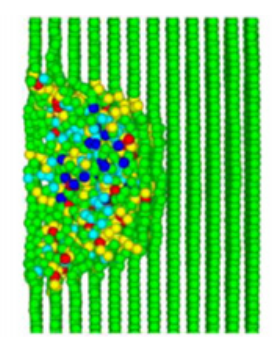

(a) $\mathrm{t}=0.4 \mathrm{ps}$

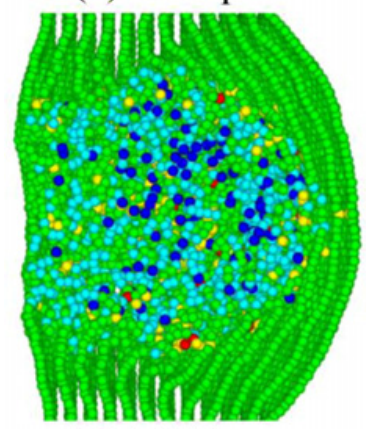

(c) $\mathrm{t}=0.8 \mathrm{ps}$

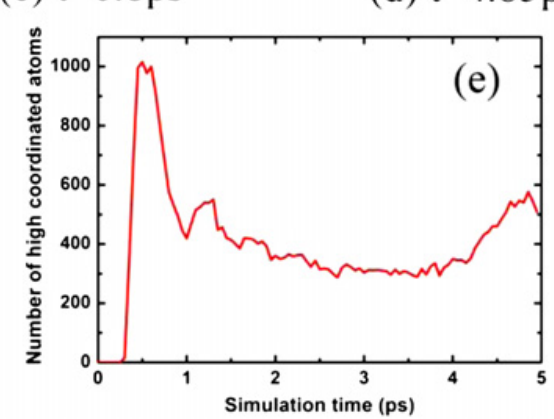

Fig. 2. $(a-d)$ Schematic for number of coordination neighbors per carbon atom in the 12-layer graphite target ( $m=1$ in blue, $m=2$ in cyan, $m=3$ in green, $m=4$ in yellow and $m>4$ in red). (e) Evolution of number of high coordinated atoms $(m \geqslant 4)$.

locity is the minimum velocity of the projectile required to perforate the target with a zero residual velocity [7]. First, we start the simulation with a high velocity so that the projectile passes through the target easily. Then by decreasing the impact velocity the residual velocity gets close to zero and we get the ballistic limit velocity. The simulation results indicate that the ballistic limit velocity increases with decreasing $n$. $V_{b l}$ (the ballistic limit velocity) is $13.0 \mathrm{~km} / \mathrm{s}$ in the case of $n=12$ (a monolithic graphite plate). But in the case of $n=1 V_{b l}$ increases to $20.0 \mathrm{~km} / \mathrm{s}, 53.85 \%$ higher than the case of $\mathrm{n}=12$. In our simulation the system with one graphene monolayer per plate has the highest penetration resistance.

We also calculated the number of coordination neighbors (denoted by $m$ ) for each carbon atom in the targets. Our simulation results for the system $n=12$ are shown in figure 2. Figures 2(a)-2(b) indicate that in the early stage of the impaction carbon atoms belong to adjacent graphene layers are compressed together and interact with each other forming many $\mathrm{sp}^{3}$ hybridized carbon atoms $(m=4)$ and other higher coordinated atoms $(m>4)$. That is to say, the target undergoes a structural phase transformation (SPT). Figure 2(e) demonstrates that the number of high coordinated atoms $(m \geqslant 4)$ reaches a 
(a)

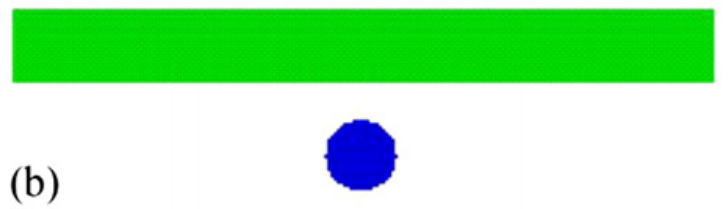

(c)

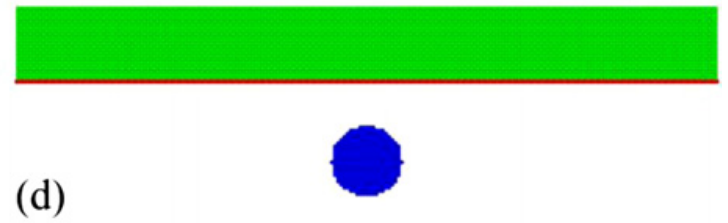

(e)

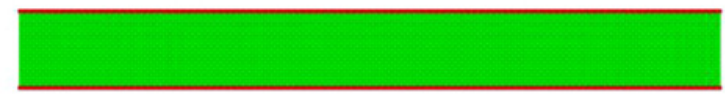

Fig. 3. Simulation models for laminated copper/graphene composites. (a) Monolithic copper plate (in green). (b) Graphene (in red) on the impact surface. (c) Graphene on the free surface. (d) Graphene between 2 thin copper plates. (e) Graphene both on the impact and free surfaces. The blue sphere is the copper projectile.

maximum at $0.5 \mathrm{ps}$. After $0.6 \mathrm{ps}$ tensile deformation takes place and the number of high coordinated atoms decreases rapidly because of reverse transformation (figures 2(c) and $2(\mathrm{e})$ ), and after $4.2 \mathrm{ps}$ there is a small rise of this number indicating that the target is rebounded from tensile deformation (figures 2(d) and 2(e)). But in the system $n=$ 1 the distance between adjacent graphene layers is large enough to avoid interactions between them. Therefore, the target undergoes only tensile deformation during the penetration process. Hence, we can speculate that the interferer between adjacent graphene layers and structural phase transformation reduce the strength and penetration resistance of the graphite target.

The penetration resistance of laminated copper/ graphene composites has also been investigated. 5 types of model have been established. One is a monolithic copper plate (figure 3(a)) with the size of $400 \times 400 \times 40 \AA^{3}$ in the $x$, $\mathrm{y}$ and $\mathrm{z}$ directions, respectively. It contains 561,672 copper atoms. The other 4 are copper/graphene composites with graphene layer on the impact (figure 3(b)) or free surface (figure 3(c)), between 2 thin copper plates (figure 3(d)), and both on the impact and free surfaces (figure 3(e)). A spherical copper cluster with a radius of $20 \AA$ (totally
2819 copper atoms) is used as the projectile. The time step used in the calculation is $1 \mathrm{fs}$. Before impacting the system has been relaxed for 20000 MD steps. We first obtain the ballistic limit velocity for the monolithic copper plate. For this purpose, we began the simulation with a low impact velocity under which a crater was formed. Then we increase the impact velocity and the depth of the crater increases too, until the copper plate has totally been perforated. Then the projectile is given this critical velocity to impact other 4 composites.

The simulation results show that under normal impact with the critical velocity the 4 composites have very different responses from each other. For the composite with graphene on the impact surface the projectile fell to pieces after impacting on the graphene layer and there formed a crater in the copper plate seen from the back surface. In the case of graphene on the free surface the projectile impacted on the copper plate forming a little crater without perforation. For the composite with graphene layer lain between 2 thin copper plates, the upper copper plate has already been perforated which is not the case for the lower plate. In the last case, in which graphene both lie on the impact and free surfaces the projectile fell to pieces and there is no crater in the copper plate. In all these cases the graphene layer has no damage at all. It revealed that the laminated copper/graphene composite with graphene layers both on the impact and free surfaces has the best penetration resistance. This phenomenon is reasonable because in this study the interaction between graphene monolayer and copper plates is described by a weak ven der Waals force which couldn't destroy the structure of graphene monolayer, hence the graphene layer could protect the copper plate since it has much higher tensile strength than any other materials.

\section{Summary}

In summary, hypervelocity impact protection properties of two different graphene based systems are investigated using molecular dynamics simulations. The first one is the spaced multilayer graphene plates. Each model contains totally 12 graphene monolayers divided into several spaced plates. The simulation results indicate that under normal impact of a spherical projectile the penetration resistance increases with decreasing number of monolayers per plate, and the system with one graphene monolayer per plate has the highest penetration resistance. The other system being studied is the laminated copper/graphene composite. The results show that under normal impact by a spherical projectile the laminated copper/graphene composite has much higher penetration resistance than the monolithic copper plate. And the composite with graphene layers both on the impact and free surfaces has the best penetration resistance.

\section{References}

1. K. S. Novoselov, A. K. Geim, S. V. Morozov, D. Jiang, Y. Zhang, S. V. Dubonos, I. V. Grigorieva, and A. A. Firsov, Science 306, 666 (2004). 
2. K. S. Novoselov, A. K. Geim, S. V. Morozov, D. Jiang, M. I. Katsnelson, I. V. Grigorieva, S. V. Dubonos, and A. A. Firsov, Nature 438, 197 (2005).

3. C. Lee, X. Wei, J. W. Kysar, and J. Hone, Science 321, 385 (2008).

4. J.-W. Jiang, J.-S. Wang, and B. Li, Phys. Rev. B 80, 113405 (2009)

5. W. Frank, D. M. Tanenbaum, A. M. van der Zande, and P. L. McEuen, J. Vac. Sci. Technol. B 25, 2558 (2007).

6. S. Dey, T. Borvik, X. Teng, T. Wierzbicki, and O. Hopperstad, Int. J. Solids. Struct. 44, 6701 (2007).
7. Alavi Nia and G. R. Hoseini, Mater. Des. 32, 1057 (2011).

8. S. Yadav and G. Ravichandram, Int. J. Impact. Eng. 28, 557 (2003).

9. M. A. Makeev and D. Srivastava, Compos. Sci. Technol. 68, 2451 (2008).

10. S. Plimpton, J. Comput. Phys. 117, 1 (1995).

11. S. J. Stuart, A. B. Tutein, and J. A. Harrison, J. Chem. Phys. 112, 6472 (2000).

12. S. M. Foiles, M. I. Baskes, and M. S. Daw, Phys. Rev. B 33, 7983 (1986).

13. M. NeeK-Amal, R. Asgari, and T. Rahimi, Nanotechnology 20, 135602 (2009). 